\title{
Efetividade do tratamento ortodôntico interceptativo com aparelho removível em crianças com mordida cruzada e mordida aberta na fase de dentadura mista
}

\author{
Effectiveness of interceptive orthodontic treatment with removable braces in children with \\ crossbite and open bite in the mixed dentition phase
}

Efectividad del tratamiento de ortodoncia interceptiva con aparatos removibles en niños con mordida cruzada y mordida abierta en la fase de dentición mixta

\section{Resumo}

A interceptação de maloclusões em estágios iniciais contribui para um crescimento equilibrado das bases ósseas. Dentre as maloclusões que devem ser corrigidas antecipadamente, destacam-se as mordidas cruzadas e mordidas abertas, nessa ordem de prioridade. Uma gama de possibilidades terapêuticas existe descritas na literatura, entre essas, o uso de aparelho removível com expansor e grade lingual. Entretanto, poucas evidências validam essa forma de tratamento, baseado nesse pressuposto o objetivo do presente estudo foi avaliar a efetividade do aparelho removível com expansor e grade lingual para correção da mordida cruzada posterior associado a mordida aberta anterior. Analisou-se as documentações ortodônticas de 148 crianças na faixa etária de 5 a 12 anos de idade que receberam tratamento entre os anos de 2009 e 2018. A efetividade ou não do tratamento foi verificada ao analisar fotografias e modelos de estudo em duas etapas de tratamento: inicial e final. Após realização de testes Shapiro-Wilk, as frequências foram comparadas pelo teste qui-quadrado e as variáveis calculadas a partir do teste Kruskal-Wallis. Foi constatado que $74 \%$ dos casos de mordida cruzada e $91,7 \%$ dos casos de mordida aberta foram corrigidos. Dessa forma pode-se concluir que a correção da mordida aberta e mordida cruzada foi conseguida com o uso desses dispositivos.

Palavras-chave: Maloclusão; Ortodontia preventiva; Mordida aberta; Dentição mista.

\begin{abstract}
The interception of malocclusions in early stages reduces the severity of the present problem, contributing to a balanced growth of bone bases. Among the malocclusions that must be corrected in advance, we highlight cross bites and open bites in that order of priority. A range of therapeutic possibilities are described in the literature, including the use of a removable appliance with expander and lingual grid. However, little evidence validates this form of treatment based on this assumption. The objective of the present study was to evaluate the effectiveness of the removable appliance with expander and lingual grid to correct posterior crossbite associated with anterior open bite. Orthodontic
\end{abstract}


documentation of 148 children aged 5 to 12 years who received treatment between 2009 and 2018 was analyzed. The effectiveness or not of the treatment was verified by analyzing photographs and study models in two stages of treatment: initial and final. After performing Shapiro-Wilk tests, the frequencies were compared using the chi-square test and the variables were calculated using the Kruskal-Wallis test. It was found that $74 \%$ of cases of crossbite and $91.7 \%$ of cases of open bite were corrected. Thus, it can be concluded that the correction of open bite and crossbite was achieved with the use of these devices.

Keywords: Malocclusion; Orthodontics, preventive; Open bite; Dentition, mixed.

\section{Resumen}

La interceptación de maloclusiones en etapas tempranas contribuye a un crecimiento equilibrado de las bases óseas. Entre las maloclusiones que deben corregirse previamente, destacan las mordidas cruzadas y las mordidas abiertas, en ese orden de prioridad. En la literatura se describe una gama de posibilidades terapéuticas, incluido el uso de un aparato removible con expansor y rejilla lingual. Sin embargo, poca evidencia valida esta forma de tratamiento, con base en este supuesto, el objetivo del presente estudio fue evaluar la efectividad del aparato removible con expansor y rejilla lingual para corregir la mordida cruzada posterior asociada con mordida abierta anterior. Se analizó la documentación ortodóncica de 148 niños de 5 a 12 años que recibieron tratamiento entre 2009 y 2018. Se verificó la efectividad o no del tratamiento mediante el análisis de fotografías y modelos de estudio en dos etapas del tratamiento: inicial y final. Después de realizar las pruebas de Shapiro-Wilk, las frecuencias se compararon mediante la prueba de chi-cuadrado y las variables se calcularon mediante la prueba de Kruskal-Wallis. Se encontró que el 74\% de los casos de mordida cruzada y el $91,7 \%$ de los casos de mordida abierta fueron corregidos. Así, se puede concluir que la corrección de mordida abierta y mordida cruzada se logró con el uso de estos dispositivos.

Palabras clave: Maloclusión; Ortodoncia preventiva; Mordida abierta; Dentición mixta.

\section{Introdução}

Maloclusão é um termo utilizado na odontologia para definir alterações no desenvolvimento craniofacial, envolvendo ossos, línguas e músculos, podendo ocasionar deformidades faciais e alterações de natureza funcional e estética, comprometendo a qualidade de vida dos acometidos (Traebert, Zanini, Nunes \& Traebert, 2020). Dentre as maloclusões que se destacam na dentição decídua e mista cita-se em especial as mordidas cruzadas (Pompei, 2005) e mordidas abertas (Sousa, Taveira \& Almeida, 2004).

A mordida aberta é caracterizada pela ausência de contato ou trespasse vertical entre os dentes; com etiologia multifatorial, podendo ser esquelética quando associada a fatores genéticos; ou dento alveolar - quando há um predomínio de fatores ambientais, como: onicofagia, interposição lingual, deglutição atípica e hábitos de sucção (Forte e Bosco, 2001; Mercadante, 2004). Alguns estudos (Lentini et al., 2014) afirmam que a prevalência de mordida aberta em crianças na dentição mista varia entre $17 \%$ a $18 \%$, no entanto, quando essa maloclusão é associada a hábitos de sucção sua prevalência aumenta para $36,3 \%$.

Já a mordida cruzada é caracterizada por um desalinhamento dos dentes, notado em oclusão cêntrica, impossibilitando que o arco maxilar oclua normalmente com o arco mandibular (Rocha Júnior, Braga e Ribeiro, 2005; Tashima, Verrastro, Ferreira, Wanderley e Guedes-Pinto 2003). A mordida cruzada não é auto corrigível com a erupção dos dentes permanentes, sendo necessário tratamento precoce com aparelho ortodôntico (Souza, Félix, Pithon \& Freitas, 2013). Fatores etiológicos ligados a condição de mordida cruzada possuem origem desconhecida, no entanto é sugerida a falha de desenvolvimento dentário, hereditariedade, retenção prolongada de dentes decíduos, extrações prematuras, traumatismos e hábitos de sucção (Moyers, 1991; Proffit e Fields, 1993).

Diversos estudos na literatura avaliaram a frequência de mordida cruzada nas dentições decíduas e mista, Pinheiro e Souza (2009) verificaram que 30,7\% das crianças na faixa etária de 6 a 10 anos apresentava algum tipo de oclusopatia, sendo que desse valor 9,5\% apresentaram mordida cruzada anterior e 6,7\% mordida cruzada posterior. Silva Filho (2003) também constatou em seu estudo que 73,26\% dos escolares (2016 alunos) na faixa etária de 3 e 6 anos de idade apresentavam alguma oclusopatia e desses $11,65 \%$ eram mordida cruzada posterior unilateral e $0,79 \%$ mordida cruzada posterior unilateral associada a mordida cruzada anterior (Silva Filho, Silva, Rego \& Capelozza Filho, 2003). 
Independente da condição de mordida, seja ela cruzada ou aberta, para obtenção de uma resposta efetiva do tratamento ortodôntico com a interceptação nas fases de dentição decídua e mista, é necessário um correto diagnóstico e um plano de tratamento apropriado, que varia desde apenas a remoção de um hábito deletério, até um tratamento mais técnico como utilização de aparelhos removíveis. Importante ressaltar que a regressão da maloclusão só é possível, se houver associação à colaboração do paciente quanto ao uso contínuo do aparelho ortodôntico (Souza, Luz, Freitas \& Pithon, 2010).

Partindo desse pressuposto pergunta-se, é possível corrigir mordida cruzada associada a mordida aberta com o uso de aparelho removível? Diante desse questionamento se propôs a realização do presente estudo.

\section{Metodologia}

Trata-se de um estudo quantitativo, transversal retrospectivo e descritivo, conduzido de acordo com os princípios exigidos pelo Comitê de Ética e Pesquisa (CEP), no qual, obteve parecer favorável, CAAE 91703118.8.0000.0055.

Foram analisadas informações de 204 fichas clínicas do arquivo de uma clínica escola, no período entre 2009 e 2018. Todas as fichas se tratavam de pacientes com a presença de maloclusão que foram tratados com uso de aparelho ortodôntico removível (AOR).

O cálculo amostral apontou a necessidade de $\mathrm{n}=134$ crianças, utilizando uma margem de erro amostral de $5 \%$ e um nível de confiança de 95\%. A partir desta avaliação, adotou-se para o estudo a variável dependente mordida cruzada (MC) e mordida aberta (MA). O critério de inclusão adotado para a pesquisa foi: crianças entre 5 e 12 anos de idade com necessidade de tratamento ortodôntico e que nunca tivesse sido submetidas a tratamento ortodôntico prévio. Os critérios de exclusão empregados foram: problemas de preenchimento de fichas clínicas e documentação ortodôntica, utilização de outro meio de tratamento que não fosse a utilização de AOR, pacientes especiais ou sindrômicos com dificuldade de colaboração no tratamento, crianças com alterações esqueléticas severas, ou seja, que tinham indicação de aparelhos fixos e ortopédicos, e aquelas com necessidade de adequação do meio bucal.

Para avaliação da efetividade dos tratamentos um pesquisador, previamente calibrado por um especialista em Ortodontia $(\mathrm{K}>0,94)$, avaliou os modelos de estudo e as fotografias da documentação inicial e final de cada paciente. $\mathrm{O}$ tempo de tratamento considerado para este estudo variou entre 6 e 24 meses. Os casos considerados finalizados sem correção do problema, fizeram parte do estudo quando se encontravam dentro desse período de tratamento ou os casos de crianças que não colaboraram com o uso do aparelho. As crianças foram fotografadas e moldadas semestralmente. Além disso, foi aplicado um questionário aos responsáveis, possibilitando analisar os hábitos bucais durante o uso de AOR, a proservação dos casos após o tratamento e a confirmação de outros dados relacionados a maloclusão.

Para a correção da variável Mordida Aberta foi padronizado e utilizado o aparelho ortodôntico removível tipo placa de Hawley com grade palatina, associada ou não a parafuso expansor com cobertura oclusal, nos casos com alterações transversais associadas (Figura 1). Nos casos da variável mordida cruzada posterior foi padronizado e utilizado o aparelho ortodôntico removível placa de Hawley com parafuso expansor e cobertura oclusal, e nos casos de cruzamento em dentes anteriores foram adicionadas molas digitais (Figura 2 A-C). 
Figura 1 - Aparelho ortodôntico removível Placa de Hawley com grade palatina, e cobertura oclusal.

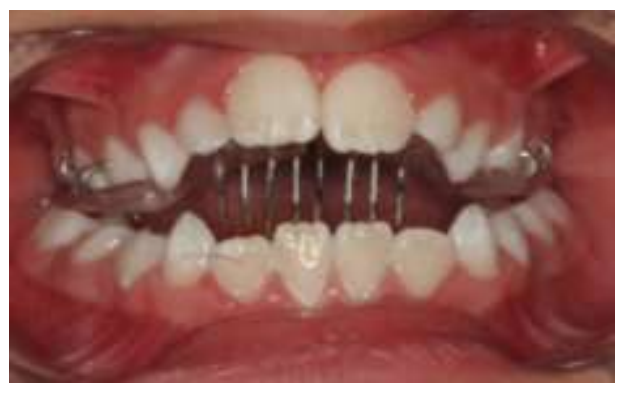

Fonte: Autores.

Figura 2 - Aparelho ortodôntico removível (AOR). A) Placa de Hawley com expansor. B) Placa de Hawley com expansor e molas digitais. C) Placa de Hawley com expansor e cobertura oclusal.

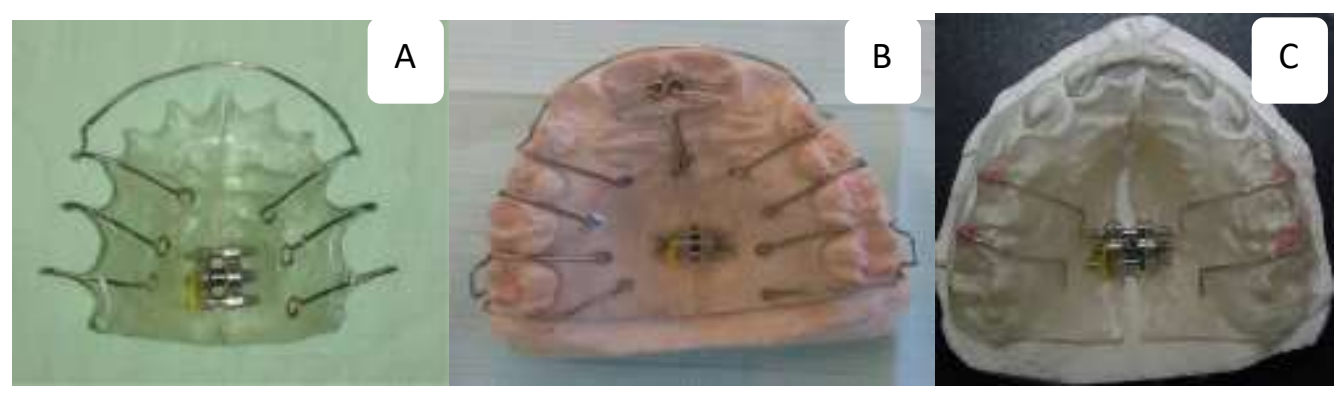

Fonte: Autores.

Foram considerados 2 padrões avaliativos para analisar a efetividade dos tratamentos das mordidas abertas e mordidas cruzadas através da utilização de AOR. Nas mordidas cruzadas, foi observado o desalinhamento dos dentes dos arcos antagônicos em oclusão cêntrica, distinguindo em duas classes: tratamento não corrigido quando não identificava diferença entre o antes e depois, e o arco maxilar permanecia não ocluindo normalmente com o arco mandibular; e tratamento corrigido quando observou correção do desalinhamento e o arco maxilar passou a ocluir normalmente com o arco mandibular (Figuras 3 A-B). Nos casos de mordida aberta anterior a análise foi baseada no distanciamento das bordas incisais dos arcos antagônicas, também distinguido em duas classes: tratamento não corrigido, quando não identificava diferença entre o antes e o depois; e tratamento corrigido, quando observou um transpasse vertical positivo (Figura 4 A-B). 
Figura 3 - Modelos de estudo em gesso de paciente com mordida cruzada anterior e posterior corrigida. A) Inicial. B) Final

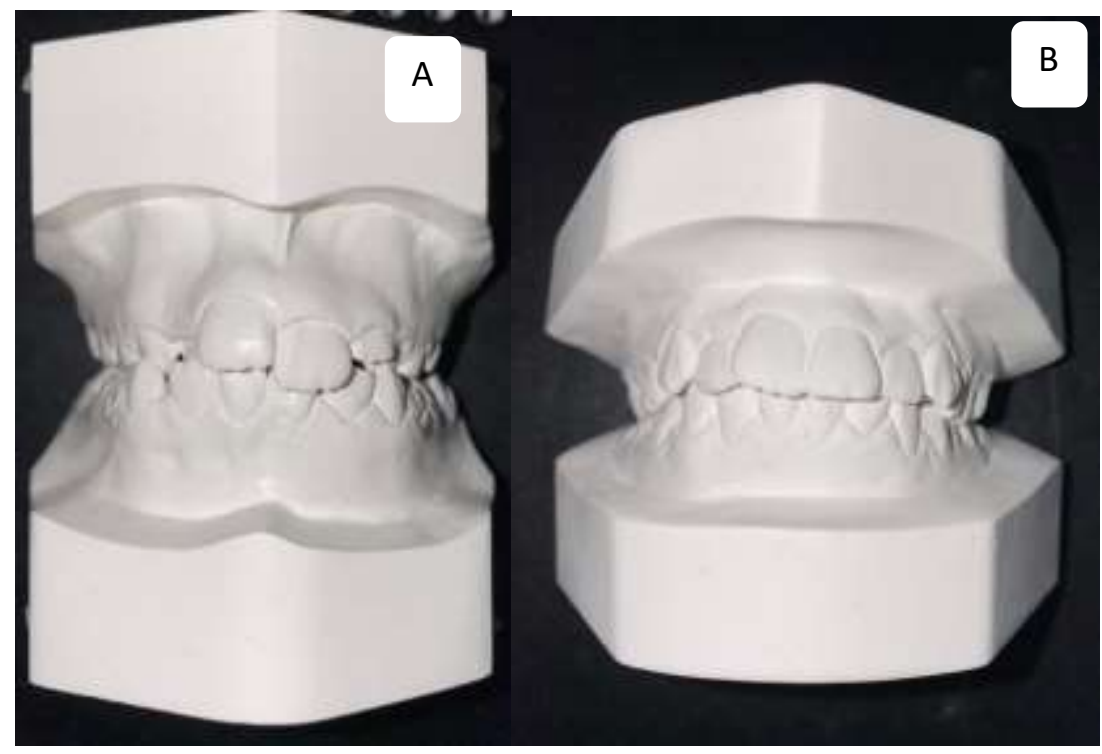

Fonte: Autores.

Figura 4 - Modelos de estudo em gesso de paciente com mordida aberta corrigida. A) Inicial. B) Final

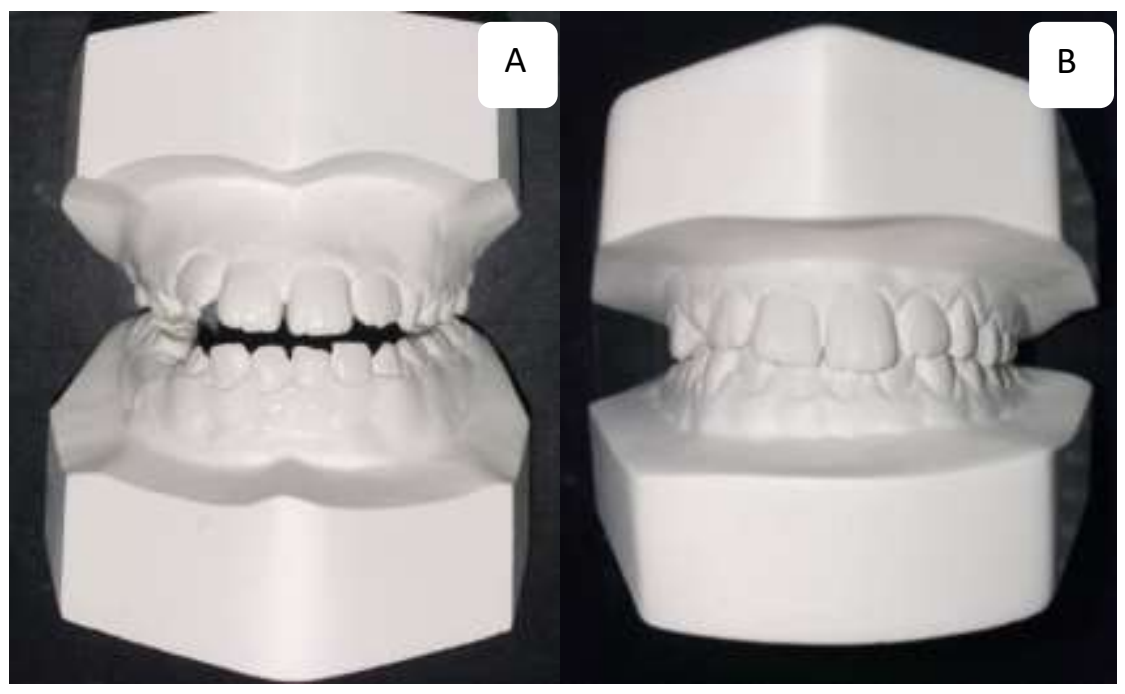

Fonte: Autores.

\section{Resultados}

Foram examinadas 204 crianças e delas obteve-se 100 casos de mordida cruzada e 48 casos de mordida aberta (5-12 anos), tratados com aparelhos ortodônticos removíveis. Houve distribuição similar entre os sexos. A mediana da idade foi de 9 anos $(\mathrm{AIQ}=1,0)$ e de tempo de tratamento foi de 8 meses $(\mathrm{AIQ}=8,0)$. (Tabela 1$)$ 
Tabela 1. Características demográficas e clínicas dos casos incluídos no estudo.

\begin{tabular}{ll}
\hline Variável & $\mathrm{n}(\%) /$ mediana \pm amplitude interquartil \\
\hline Sexo & $76(51,35 \%)$ \\
Feminino & $72(48,65 \%)$ \\
Masculino & $9 \pm 1$ \\
Idade (anos) & $8 \pm 8$ \\
Tempo de tratamento (meses) & \\
Tipo de mordida & $48(32,4 \%)$ \\
Aberta & $52(35,1 \%)$ \\
Cruzada anterior & $28(19,0 \%)$ \\
Cruzada posterior & $20(13,5 \%)$ \\
Cruzada anterior - posterior
\end{tabular}

Fonte: Autores.

O Gráfico 1 representa os hábitos deletérios atuante nas crianças avaliados mediante questionário para o padrão de mordida aberta.

Gráfico 1 - Gráfico de prevalência de hábitos deletérios em crianças com mordida aberta associada ao sexo.

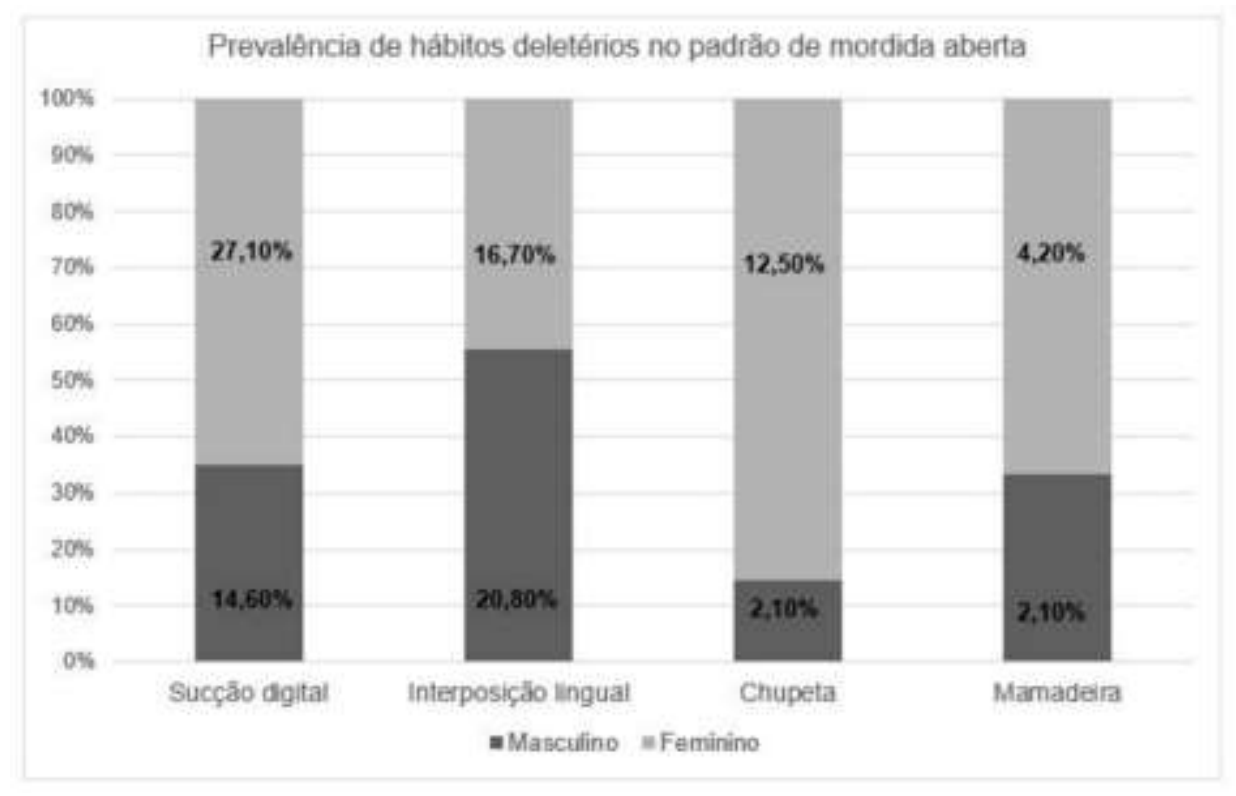

Fonte: Autores.

Não foi observado diferença significativa na efetividade do tratamento, em relação ao sexo e tipo de mordida cruzada (Tabela 2), assim verificou-se que em, aproximadamente, três quartos dos casos (74\%) o tratamento empregado promoveu correção do quadro clínico, sendo que em $26 \%$ dos pacientes não foi possível corrigir a disfunção. De forma similar, a idade dos pacientes e o tempo de tratamento não diferiu significativamente, em função da efetividade do tratamento (Figura 5). 
Tabela 2. Efetividade do tratamento em pacientes com mordida cruzada, tratados com aparelhos ortodônticos removíveis, de acordo com o sexo e o tipo de mordida.

\begin{tabular}{|c|c|c|c|c|c|}
\hline \multirow[t]{2}{*}{ Variável } & \multicolumn{2}{|c|}{ Corrigido } & \multicolumn{2}{|c|}{ Não corrigido } & \multirow[t]{2}{*}{ *p - valor } \\
\hline & $\mathrm{n}$ & $\%$ & $\mathrm{n}$ & $\%$ & \\
\hline \multicolumn{6}{|l|}{ Sexo } \\
\hline Feminino & 34 & $72,3 \%$ & 13 & $27,7 \%$ & 0,721 \\
\hline Masculino & 40 & $75,5 \%$ & 13 & $24,5 \%$ & \\
\hline \multicolumn{6}{|l|}{ Tipo de mordida } \\
\hline Cruzada anterior & 36 & $69,2 \%$ & 16 & $30,8 \%$ & \\
\hline Cruzada posterior & 20 & $71,5 \%$ & 8 & $28,6 \%$ & 0,185 \\
\hline Cruzada anterior - posterior & 18 & $90 \%$ & 2 & $10,0 \%$ & \\
\hline
\end{tabular}

*Teste qui-quadrado. Fonte: Autores.

Figura 5 - Idade (A) e tempo de tratamento (B), de acordo com a efetividade do tratamento em pacientes com mordida cruzada, tratados com aparelhos ortodônticos removíveis. As colunas representam as medianas e as barras de erros as amplitudes interquartis.

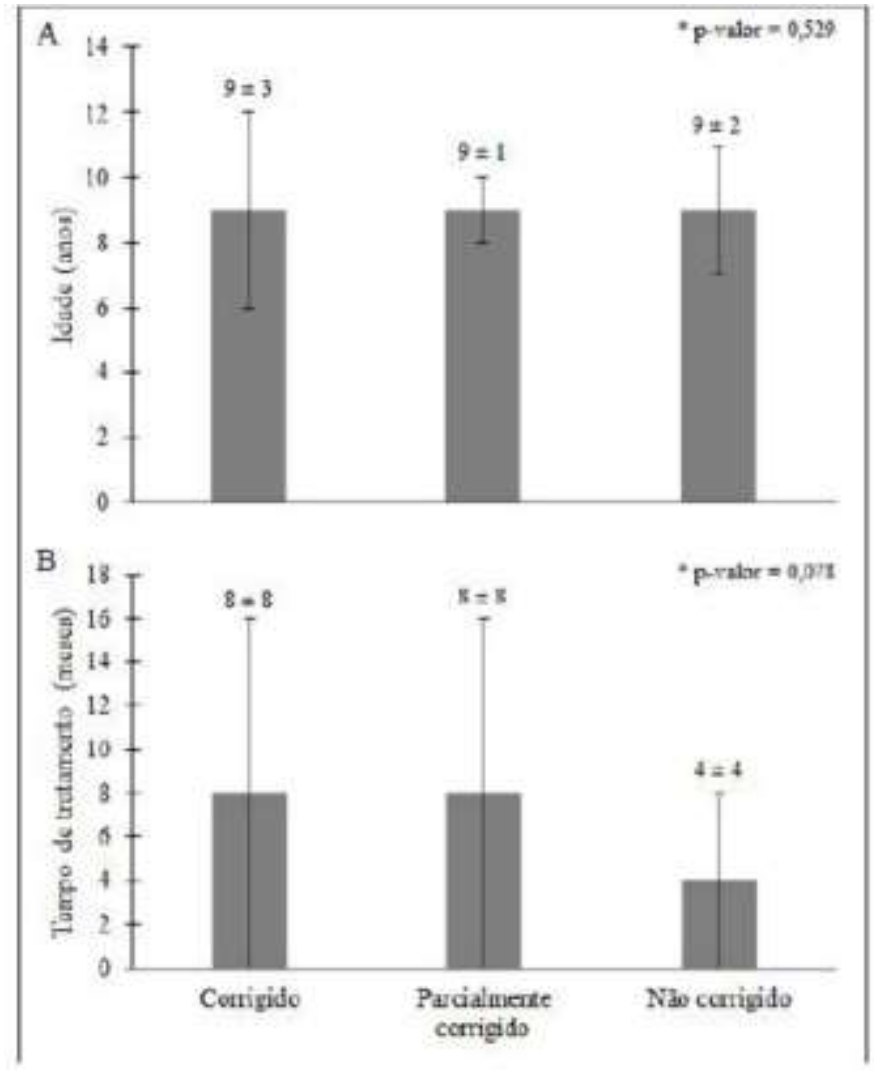

* Teste Kruskal-Wallis. Fonte: Autores. 
Assim como na mordida cruzada, na mordida aberta também não foi observado diferença significativa na efetividade do tratamento, em relação ao sexo.

Tabela 3. Efetividade do tratamento em pacientes com mordida aberta, tratados com aparelhos ortodônticos removíveis, de acordo com o sexo e o tipo de mordida.

\begin{tabular}{|c|c|c|c|c|}
\hline \multirow[t]{2}{*}{ Variável } & Corrigido & \multicolumn{2}{|c|}{ Não corrigido } & \multirow[t]{2}{*}{ *p - valor } \\
\hline & $\mathrm{n} \quad \%$ & $\mathrm{n}$ & $\%$ & \\
\hline \multicolumn{5}{|l|}{ Sexo } \\
\hline Feminino & $89,66 \%$ & 3 & $10,34 \%$ & 0,533 \\
\hline Masculino & $94,73 \%$ & 1 & $5,3 \%$ & \\
\hline \multicolumn{5}{|c|}{ Tipo de mordida } \\
\hline Aberta & $91,7 \%$ & 4 & $8,33 \%$ & \\
\hline
\end{tabular}

*Teste qui-quadrado. Fonte: Autores.

Nos Gráficos 2 e 3 é possível avaliar a efetividade do tratamento de mordida cruzada e mordida aberta, respectivamente, sem distinção de sexo, classificando os tratamentos das 148 crianças participantes da pesquisa em corrigido e não corrigido.

Gráfico 2 - Efetividade do tratamento em pacientes com mordida cruzada, tratados com aparelhos ortodônticos removíveis.

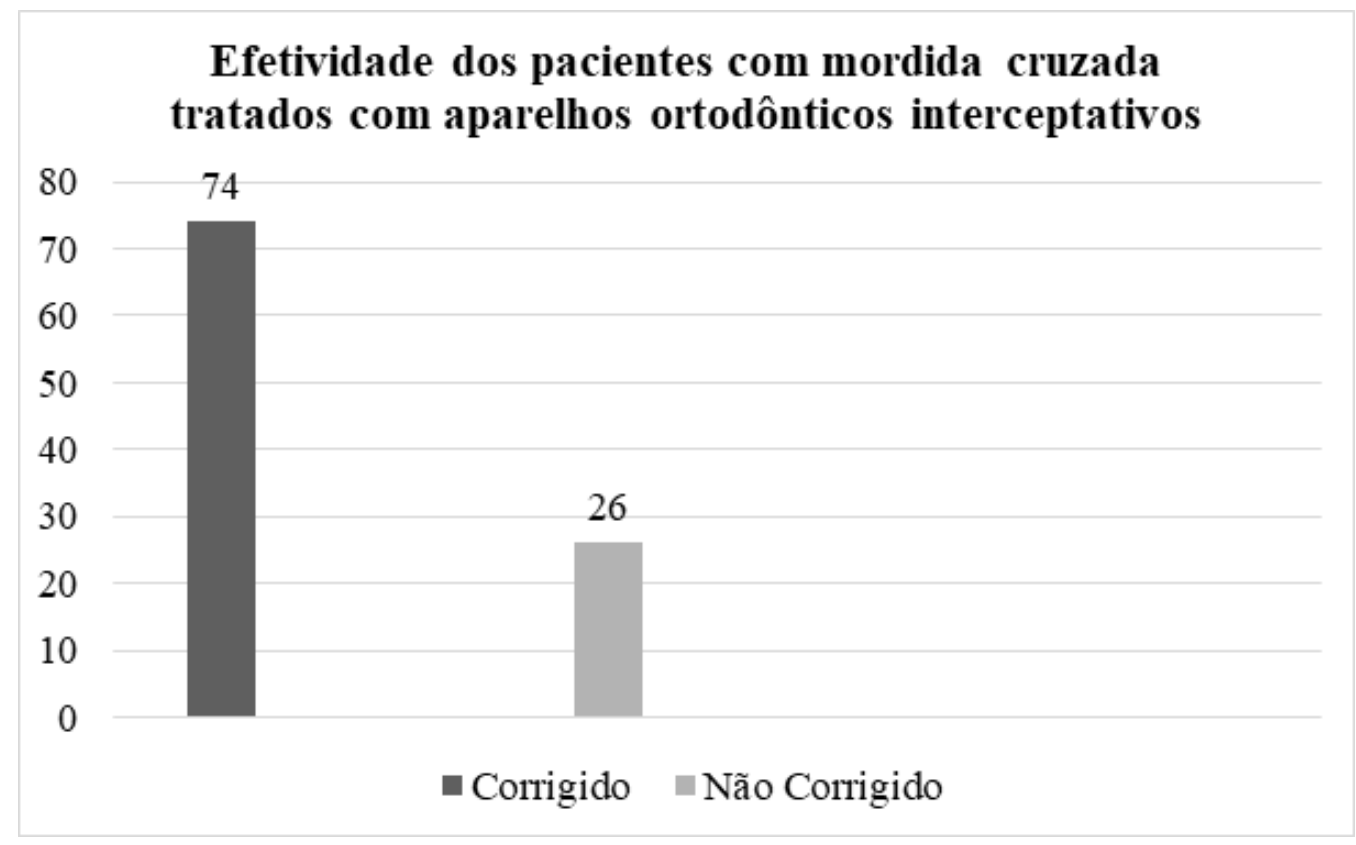

Fonte: Autores. 
Gráfico 3 - Efetividade do tratamento em pacientes com mordida aberta, tratados com aparelhos ortodônticos removíveis.

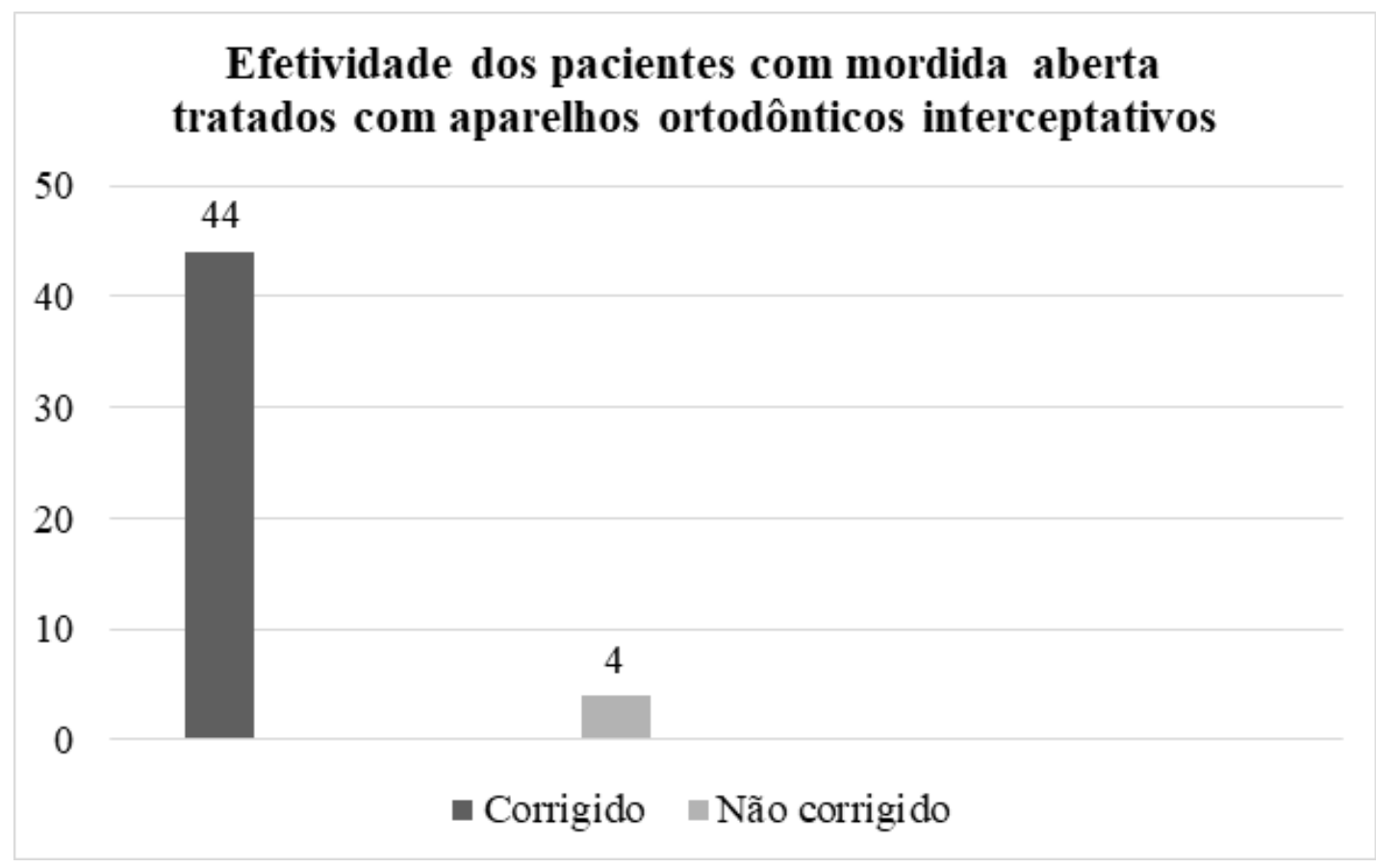

Fonte: Autores.

\section{Discussão}

\section{Mordida Cruzada}

Estudos epidemiológicos com enfoque na prevalência de maloclusões nas dentições decídua e mista têm sido realizados ao longo dos anos. Thomazine e Imparato (2000) avaliaram 525 crianças de ambos os sexos, entre 6 e 9 anos de idade, e observaram que 34,10\% eram portadoras de algum tipo de maloclusão. Desses, 20,57\% do total das crianças avaliadas apresentavam mordida cruzada. Outro estudo realizado por Souza, Luz, Freitas e Pithon (2010), na cidade de Jequié/BA, evidenciou que 30,7\% dos escolares entre 6 e 10 anos de idade, possuíam algum tipo de maloclusão, onde 9,5\% apresentavam mordida cruzada anterior e $6,7 \%$ mordida cruzada posterior.

Mediante tais dados, é possível perceber a consonância da prevalência dos estudos citados, que variam sem grandes discrepâncias, com a clínica escola em análise, na qual, dos 148 pacientes encaminhados para tratamento ortodôntico, 67,56\%, possuíam mordida cruzada, sem disparidade entre os sexos.

Nesse estudo quanto a variável de mordida, destacou-se a mordida cruzada anterior, expressa em $35,1 \%$ dos casos avaliados (Tabela 1), embora a literatura mostre que a prevalência de mordida cruzada posterior seja significativamente superior às demais (Silva Filho, Silva, Rego e Capelozza Filho, 2003; Souza, Luz, Freitas \& Pithon, 2010; Woitchunas, Busato, Tocheto \& Brockstedt, 2001).

Para o tratamento das variadas maloclusões, que inclui mordida cruzada, diversos autores preconizam o diagnóstico e interceptação nas dentaduras decídua e mista (Barich, 1952; Castaner, 2006; Tashima, Verrastro, Ferreira, Wanderley \& Guedes-Pinto, 2003), período equivalente com dos pacientes tratados nesta pesquisa (5-12 anos), em que as respostas fisiológicas são mais favoráveis diante da bioelasticidade óssea existente, o que colabora significativamente para o reequilíbrio do sistema estomatognático e minimiza a necessidade de tratamentos futuros de maior complexidade na dentadura permanente (Dutra et al., 2004).

Embora análises da efetividade dos tratamentos interceptativos a curto e longo prazo sejam escassos, a literatura traz um estudo realizado por King e Brudvik (2010), que, diferente desse estudo, utilizaram como metodologia a comparação de 
modelos de gesso de um grupo controle com um grupo interceptativo, cujas avaliações foram feitas através do índice de complexidade, desfecho e necessidade (ICON).

Para o grupo controle foram coletados modelos de gesso arquivados da década de 70 de pacientes não tratados durante a dentadura mista, enquanto que para o grupo interceptativo utilizaram modelos de pacientes recentes tratados precocemente. Diante da metodologia adotada categorizaram os resultados das análises do grupo interceptativo em melhoria mínima (22,6\%), moderada $(21,1 \%)$ e substancial $(17,3 \%)$, e perceberam que o grupo tratado obteve melhora na estética, mordida cruzada, apinhamento maxilar e mordida aberta anterior, enquanto o grupo controle não apresentou melhora em nenhuma categoria, mas sim uma piora significativa do apinhamento. Frente aos resultados, concluíram que a falta de intervenção não provoca significativas alterações das maloclusões durante a dentadura mista, mas por outro lado, ratificaram a importância da intervenção no sentido de reduzir a complexidade e necessidade.

$\mathrm{O}$ resultado da pesquisa desses autores diverge quanto à melhoras significativas quando confrontado aos resultados de um estudo realizado por al Nimri e Richardson (2000) numa comunidade da Irlanda do Norte, em que 94\% das crianças tratadas obtiveram resultados satisfatórios, com apenas $2 \%$ de agravo; e do presente trabalho, visto que obteve-se uma melhora significativa em 74\% dos casos (Gráfico 2), no entanto converge quanto à importância da intervenção no sentido de reduzir a complexidade e necessidade da maloclusão. Essa divergência pode ser justificada pela inclusão do grupo controle no estudo de King e Brudvik (2010), que difere da metodologia abordada nas outras pesquisas.

Nos casos de mordida cruzada posterior em análise neste trabalho, os tratamentos foram realizados com aparelho removível com parafuso expansor, onde alcançou-se uma melhoria de 71,5\% dos casos. Quando não há cooperação dos pacientes no uso do aparelho removível, outro meio de expansão lenta usado para correção da mordida cruzada posterior é o quadri-hélice, em que Godoy, Godoy-Bezerra e Rosenblatt (2011) num estudo de comparação entre os dispositivos removíveis e fixos, confirma que o sucesso dos tratamentos é semelhante, no entanto o número de complicações é maior com o aparelho quadri-hélice devido à dificuldade de higienização do meio bucal e irritação aos tecidos adjacentes.

Em relação ao tempo de tratamento, nessa pesquisa, não foram encontrados registros na literatura que destacassem a associação do tempo com a efetividade do tratamento, porém, os casos não corrigidos nesse estudo (26\%) apresentaram um tempo de tratamento inferior quando comparado aos casos que foram corrigidos (Tabela 2), ou seja, os pacientes que descontinuaram o tratamento não obtiveram respostas satisfatórias. Embora, a adesão seja alta pela qualidade do tratamento oferecida pelo serviço público em uma clínica escola, essa descontinuidade pode ser explicada por fatores que interferem diretamente nos resultados, como falta de motivação e reforço positivo por parte dos responsáveis, condições socioeconômicas para estar presente em todas as consultas, e intervalos entre um semestre e outro, que resulta em desadaptação dos aparelhos e por consequência a não utilização dos mesmos. Assim, é observado que mesmo com alta adesão a colaboração por partes dos pacientes ainda é a maior dificuldade durante o tratamento.

\section{Mordida aberta}

Estudos mostram que há uma associação estatisticamente significativa entre as maloclusões e os hábitos deletérios (Barreto, Souza, Freitas \& Pithon, 2012; Carvalho, Carvalho, Forte, Aragão \& Costa, 2009; Chen, Xia, Ge, 2015; Corrêa-Faria, Ramos, Martins Junior, Andrade \& Marques, 2014). Nesta pesquisa foi identificado (Gráfico 1), dentre os 48 casos de mordia aberta anterior, a presença de interposição ou deglutição atípica com 18 (37,5\%) dos casos, e hábitos de sucção, tais como chupeta com 7 (14,6\%), mamadeira com 3 (6,3\%) e dedo com 20 (41,7\%). O resultado diverge quando comparado ao de Carvalho, Carvalho, Forte, Aragão e Costa, 2009 (2009) e Miotto, Cavalcante, Godoy, Campos e Barcellos (2014), que mostraram uma diferença substancial entre os hábitos mais prevalentes e o de suçãa digital. Essa diferença pode ser 
justificada pela diferença de metodologias, e por ser uma coleta de dado dependente da compreensão e memória dos responsáveis ao responderem esta pesquisa.

Pesquisas afirmam que é a dentição mista (Carvalho, Carvalho, Forte, Aragão \& Costa, 2009; Góes, Araújo, Góes e Jamelli, 2013; Morais, Mota \& Amorim, 2014) o período mais propício para remoção do HOD e correção das maloclusões. A retirada do hábito pode ser realizada através de condicionamento psicológico ou com o uso de dispositivos de auxilio, como o aparelho ortodôntico removível, que em casos de MA apresenta grade palatina (Reis, Pinheiro \& Malafaia, 2007). Os aparelhos atuam de forma interceptativa e funcional, eliminando o hábito e/ou recondicionando a língua (Proffit, 2013). Deste modo, as crianças que possuíam mordida aberta foram avaliadas, analisando a efetividade do tratamento interceptativo do aparelho ortodôntico removível com grade palatina, no qual foi visto grande eficácia quando comparados início e final do tratamento.

\section{Conclusão}

Conclui-se com a realização desse estudo que os aparelhos ortodônticos removíveis do tipo grade lingual e expansor são efetivos para correção da mordida aberta e a mordida cruzada respectivamente, apesar da necessidade de colaboração das crianças em fase de dentição mista com o uso do aparelho.

\section{Referências}

Al Nimri, K., \& Richardson, A. (2000). Interceptive orthodontics in the real world of community dentistry. International journal of paediatric dentistry, 10(2), 99-108.

Barich, F. T. (1952). Treatment in the mixed dentition period. Am. J. Orthodont. Dentofacial Orthop 38 (6), $25-33$

Barreto, L. B., Souza, R. A., Freitas, L. M., \& Pithon, M. M. (2012). Association between breastfeeding and deleterious oral habits with development of malocclusions in pediatric dental patients. Int J Exp Dental Sci, 1(2), 61-6.

Carvalho, C. M., da Costa Carvalho, L. F. P., Forte, F. D. S., do Socorro Aragão, M., \& da Costa, L. J. (2009). Prevalência de mordida aberta anterior em crianças de 3 a 5 anos em Cabedelo/PB e relação com hábitos bucais deletérios. Pesquisa Brasileira em Odontopediatria e Clínica Integrada, 9(2), 205-210.

Castañer Peiro, A. (2006). Ortodoncia interceptiva: Necesidad de diagnóstico y tratamiento temprano en las mordidas cruzadas transversales. Medicina Oral, Patología Oral y Cirugía Bucal (Internet), 11(2), 210-214.

Chen, X., Xia, B., \& Ge, L. (2015). Effects of breast-feeding duration, bottle-feeding duration and non-nutritive sucking habits on the occlusal characteristics of primary dentition. BMC pediatrics, 15(1), 1-9.

Corrêa-Faria, P., Ramos-Jorge, M. L., Martins-Júnior, P. A., Vieira-Andrade, R. G., \& Marques, L. S. (2014). Malocclusion in preschool children: prevalence and determinant factors. European Archives of Paediatric Dentistry, 15(2), 89-96.

Dutra, A. L. T., Cardoso, A. C., Locks, A., \& Bezerra, A. C. B. (2004). Assessment of treatment for functional posterior cross-bites in patients at the deciduous dentition phase. Brazilian dental journal, 15(1), 54-58.

Forte, F. D. S., \& Bosco, V. L. (2001). Prevalência de mordida aberta anterior e sua relação com hábitos de sucção não nutritiva. Pesqui. bras. odontopediatria clín. integr, 3-8.

Godoy, F., Godoy-Bezerra, J., \& Rosenblatt, A. (2011). Treatment of posterior crossbite comparing 2 appliances: a community-based trial. American Journal of Orthodontics and Dentofacial Orthopedics, 139(1), e45-e52.

Góes, M. P. S. D., Araújo, C. M. T., Góes, P. S. A., \& Jamelli, S. R. (2013). Persistência de hábitos de sucção não nutritiva: prevalência e fatores associados. Revista Brasileira de Saúde Materno Infantil, 13(3), 247-257.

King, G. J., \& Brudvik, P. (2010). Effectiveness of interceptive orthodontic treatment in reducing malocclusions. American journal of orthodontics and dentofacial orthopedics, 137(1), 18-25.

Lentini Oliveira, D. A., Carvalho, F. R., Rodrigues, C. G., Ye, Q., Hu, R., Minami Sugaya, H., \& Prado, G. F. (2014). Tratamento ortodôntico e ortopédico para mordida aberta anterior em crianças. Cochrane Database of Systematic Reviews, (9).

Mercadante, M. M. N. (2004). Hábitos em Ortodontia. In: Ferreira FV. Ortodontia: diagnóstico e planejamento clínico. São Paulo: Artes Médica; p.253-79.

Miotto, M. H. M. D. B., Cavalcante, W. S., Godoy, L. M., Campos, D. M. K. D. S., \& Barcellos, L. A. (2014). Prevalência de mordida aberta anterior associada a hábitos orais deletérios em crianças de 3 a 5 anos de Vitória, ES. Revista CEFAC, 16(4), 1303-1310.

Morais, S. P. T. D., Mota, E. L. A., \& Amorim, L. D. A. (2014). Fatores associados à incidência de maloclusão na dentição decídua em crianças de uma coorte hospitalar pública do nordeste brasileiro. Revista Brasileira de Saúde Materno Infantil, 14(4), 371-382. 
Research, Society and Development, v. 10, n. 2, e19110212433, 2021

(CC BY 4.0) | ISSN 2525-3409 | DOI: http://dx.doi.org/10.33448/rsd-v10i2.12433

Moyers, E. R, Riolo, M. L. (1991). Tratamento precoce. In Moyers (4a ed.). Guanabara Koogan.

Pinheiro, S. M. S., \& Souza, R. A. D. (2009). Assistência ortodôntica no serviço público-fase um: frequência de alterações oclusais decorrentes de hábitos orais deletérios em escolares de Jequié-BA. Ortho Sci., Orthod. sci. pract, 729-734.

Pompei, V. T. M., Carvalho, A. S. D., Cunha, F. L., \& Pompei Filho, H. (2005). Avaliação da assimetria facial em indivíduos com mordida cruzada posterior por meio de fotografias frontais. Ortodontia, 337-344.

Proffit, W. R. (2013). Ortodontia Contemporânea5: Ortodontia Contemporânea. Elsevier Health Sciences.

Proffit, W. R., \& Fields, H. W. (1993). O planejamento do tratamento ortodôntico: da lista de problemas ao plano específico. PROFFIT, WR et al. Ortodontia contemporânea, 2, 170-205.

Reis, M. J., Pinheiro, C. N., \& Malafaia, M. (2007). Tratamento da mordida aberta anterior: relato de caso clínico. Rev. Clín. Ortod. Dent. Press, 88-96.

Rocha Júnior, J. F., Braga e Silva, A. C. N., \& Ribeiro, D. O. (2005). Interceptação ortodôntica na dentadura decídua. JBP rev. Ibero-am. odontopediatr. odontol. bebê, 67-76.

Silva Filho, O. G. D., Silva, P. R. B., Rego, M. V. N. N. D., \& Capelozza Filho, L. (2010). Epidemiologia da mordida cruzada posterior na dentadura decídua. Revista Íbero-americana de Odontopediatria \& Odontologia de Bebê, 6(29).

de Sousa, F. R. N., Taveira, G. S., de Almeida, R. V. D., \& Padilha, W. W. N. (2004). O aleitamento materno e sua relação com hábitos deletérios e maloclusão dentária. Pesquisa Brasileira em Odontopediatria e Clínica Integrada, 4(3), 211-216.

Souza, R. A. D., Félix, M. F. G. R., Pithon, M. M., \& Freitas, L. M. A. D. (2013). Tratamento ortodôntico com aparelho removível no serviço público em paciente com mordida cruzada e assimetria facial: relato de caso. Ortho Sci., Orthod. sci. pract, 89-94.

Tashima, A. Y., Verrastro, A. P., Ferreira, S. L. M., Wanderley, M. T., \& Guedes-Pinto, E. (2003). Tratamento ortodôntico precoce da mordida cruzada anterior e posterior: relato de caso clínico. J Bras Odontopediatr Odontol Bebê, 6(29), 24-31.

Thomazine, G. D., \& Imparato, J. C. P. (2000). Prevalência de mordida aberta e mordida cruzada em escolares da rede municipal de Campinas. JBP, j. bras. odontopediatr. odontol. bebê, 29-37.

Traebert, E., Zanini, F. A., Nunes, R. D., \& Traebert, J. (2020). Nutritional and non-nutritional habits and occurrence of malocclusions in the mixed dentition. Anais da Academia Brasileira de Ciências, 92(1).

Woitchunas, D. R., Busato, C., Tocheto, L. R., \& Brockstedt, R. H. (2001). Mordidas cruzadas anteriores: diagnóstico e tratamento da pseudoclasse III-relato de um caso clínico. Revista da Faculdade de Odontologia-UPF, 6(2). 